\title{
The Beatles in Italy. Per una ricerca sulla storia della popular music e i lunghi anni Sessanta
}

\author{
di Ferdinando Fasce
}

Lug 3, 2019 | In evidenza, La colonna sonora di una generazione $\mid \underline{0 \mid}$

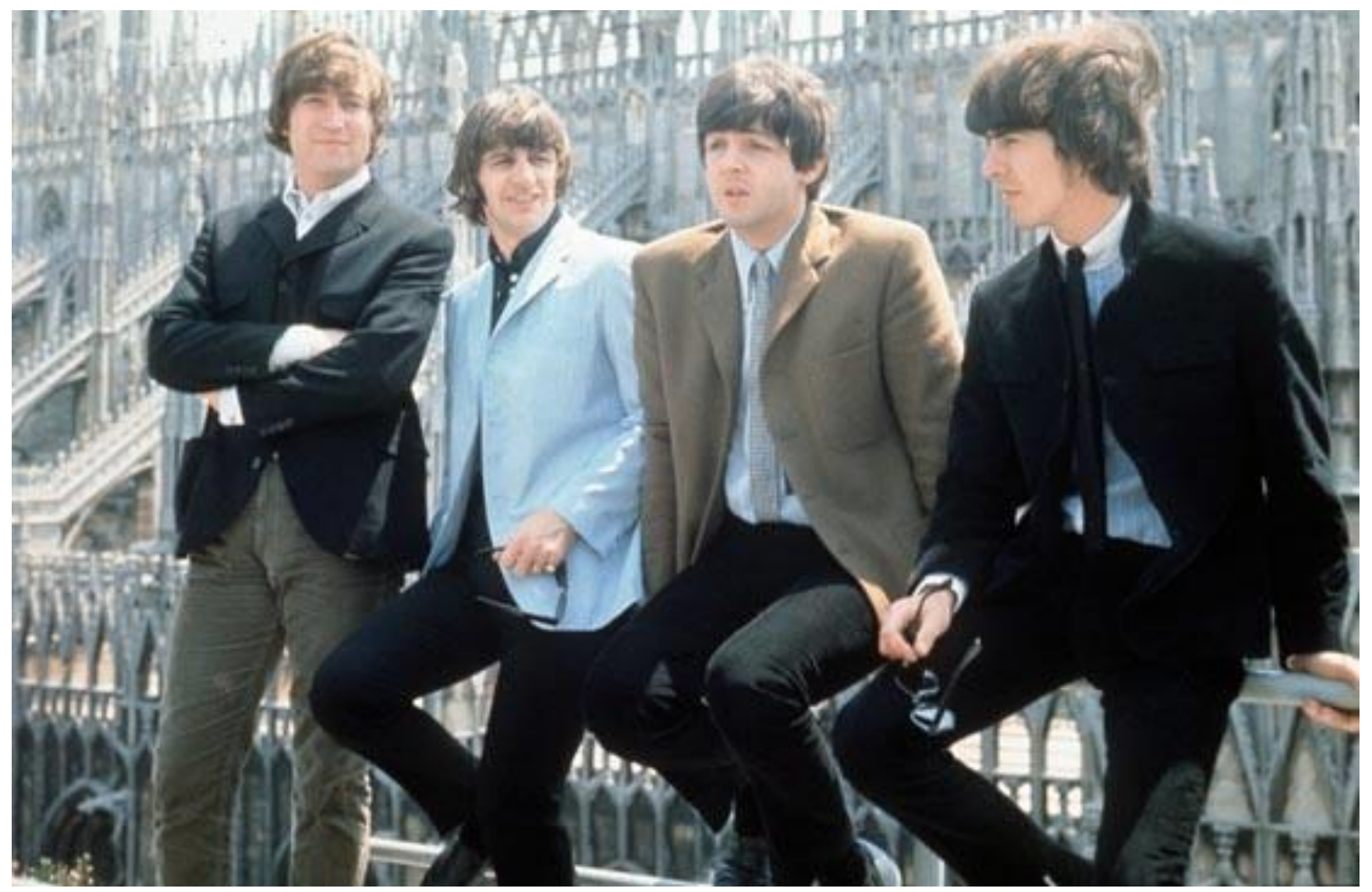

$\underline{\text { I Beatles sul tetto del Duomo di Milano durante la tournée italiana del 1965. Foto di pubblico }}$ $\underline{\text { dominio }}, \underline{\text { Collegamento }}$

\begin{abstract}
«Chiedi chi erano i Beatles», recita un brano musicale di diversi anni fa. Ma rispondere al quesito non è una cosa facile. Anche a proposito della breve tournée italiana dei Beatles del giugno 1965, oggetto finora di carrellate di impronta giornalistica, ricche di aneddoti e curiosità tratte dalla pubblicistica dell'epoca e da interviste a testimoni del mondo musicale e dell'informazione. Tenendo ben presenti questi contributi, l'autore propone un riesame dell'evento alla luce delle suggestioni, di metodo e di sostanza, che provengono dalla più recente letteratura, nazionale $\mathrm{e}$ internazionale, di storia della popular music e della società. L'ottica è quella di allargare la discussione a tutto campo, al di là di steccati disciplinari o affiliazioni professionali, fra giornalisti, critici militanti, operatori musicali, musicologi, scienziati sociali e storici, della musica e non. E illuminare così quella faccia musicale del «lungo ‘68» che resta ancora in larga parte inesplorata.
\end{abstract}




\section{PREMESSA}

The Beatles in Italy è il titolo di un disco e di almeno un paio di libri, tutti e tre in qualche modo legati alla tournée italiana del gruppo del giugno 1965[1].

Il disco uscì, solo in Italia, quell'anno, immediatamente a ridosso della breve visita della band nella penisola, per sfruttarne gli echi al botteghino fonografico. «Equivoco», lo definisce una recente rassegna della produzione discografica beatlesiana. Perché «fa supporre registrazioni dal vivo», cioè dai concerti appena tenuti dal gruppo a Milano, Genova e Roma, mentre «in realtà contiene canzoni già edite in Italia (tranne quattro» [Rizzi 2009, 50].

I libri risalgono al 1999 e al 2012. Esemplificano una parte consistente della letteratura in lingua italiana sui Beatles. Sono ampie carrellate di materiali giornalistici, integrati da interviste a testimoni del mondo musicale e dell'informazione, che delineano, con dovizia di aneddoti e curiosità, la fisionomia del tour e la parabola dell'attenzione mediatica di casa nostra al gruppo nell' arco di sette anni: dalla prima scoperta del fenomeno, nella tarda estate del 1963, al suo scioglimento, nel 1970 [Fiore 1999; Brizi e Becker 2012]. L'intento delle pagine che seguono è, tenendo ben presenti questi contributi, avviare un riesame della tournée alla luce della più recente letteratura, nazionale e internazionale, di storia della popular music e della società[2]. Nell'ottica di ingaggiare una discussione a tutto campo, al di là di steccati disciplinari o affiliazioni professionali, fra giornalisti, critici militanti, operatori musicali, musicologi, scienziati sociali e storici, della musica e non.

Per dare un'idea della cornice di studi nella quale il presente intervento si inserisce, parto da uno sguardo alle principali ricostruzioni storiche dell'intera vicenda beatlesiana disponibili in italiano, per lo più traduzioni di contributi di matrice giornalistica in lingua inglese, e da un confronto con le più significative tendenze in atto a livello mondiale.

\section{BEATLES, LA STORIA, GLI STORICI}

\section{PRIMI LAVORI: HUNTER DAVIES E PHILIP NORMAN}

La prima osservazione da fare è che a livello internazionale abbiamo dovuto attendere sino alla metà degli anni novanta del Novecento per trovare un libro di storia dei Beatles con note a piè di pagina che dettagliassero le fonti [Torkelson Weber 2016]. Sino a quel momento i due volumi che avevano dominato il campo, entrambi tradotti in italiano, erano senza note e si fondavano prima di tutto su fonti orali peraltro non chiaramente individuate. Il primo era l'unica biografia autorizzata, redatta a caldo (e apparsa nel 1968, tradotta da noi due anni dopo), sulla base di un accordo col manager del complesso Brian Epstein, dal pubblicista inglese Hunter Davies. Davies vi trascrive quasi un anno e mezzo di osservazione diretta e interviste al gruppo e al suo entourage e ci consegna una fonte preziosa, ma da trattare con cautela perché incarna una visione irenistica della band come una compatta compagine saldamente incardinata attorno alla geniale coppia LennonMcCartney [Davies 1970]. Il secondo è il contributo forse ancora oggi più popolare, Shout!,apparso all'inizio degli anni ottanta per la penna di un altro giornalista d'oltre Manica, Philip Norman, che seguì i Beatles quand'erano attivi e che ne ha poi, a distanza di anni, ripercorso le tracce mediante un'ampia messe di interviste a persone a loro in vario modo legate [Norman 1981]. Diversa è però l'impostazione, in questo caso tendenzialmente lennocentrica, una visione che la forte reazione emotiva suscitata nella sfera pubblica dalla tragica scomparsa del fondatore del gruppo, avvenuta qualche mese prima dell'uscita del libro, ha ampiamente legittimato [Terkelson Weber 2016]. 


\section{MARK HERTSGAARD E BOB SPITZ}

Di abbondanti note sono invece corredati i due contributi, entrambi opera di autori statunitensi, comparsi in originale, rispettivamente, quello del giornalista Mark Hertsgaard a metà anni novanta [Hertsgaard 2002], e quello dell'ex manager musicale (Springsteen, Elton John) Bob Spitz una decina d'anni dopo [Spitz 2006], e arrivati da noi entrambi nel XXI secolo. Testimonianza dell'emergere di una visione più equilibrata della dinamica interna al gruppo, frutto della maggiore distanza dagli avvenimenti, dell'uso di nuove fonti orali e della disponibilità di nuovi materiali tratti dagli archivi EMI ad Abbey Road grazie al lavoro di scavo del pubblicista e archivista musicale Mark Lewisohn, soprattutto il libro di Spitz si segnala per la ricchezza di fonti (comprendenti, fra l'altro, una vasta selezione della pubblicistica dell'epoca) e per un primo tentativo di loro discussione critica.

\section{LA "BIOGRAFIA COLLETTIVA" DI MARK LEWISOHN}

Nessuno di questi lavori raggiunge comunque il rigore documentario e il respiro del primo e per ora unico volume, comparso nel 2013 e finora non tradotto in italiano, della biografia collettiva, prevista originariamente in tre e ora, pare, allargata a quattro, tomi, redatta dallo stesso Lewisohn. Già noto per le sue puntigliose perlustrazioni sulle sedute di registrazione e sulla cronologia delle attività multimediali dei fab four, Lewisohn, grazie al pieno accesso agli archivi EMI (anche Hertsgaard ne ha fatto uso, ma molto limitato) e all'esame delle più diverse carte inedite, da quelle scolastiche ad altre, pubbliche e private, liverpulliane e nazionali, in un migliaio di pagine distilla un'esemplare ricostruzione che segue passo passo i fab four dalla nascita alle soglie del successo, alla fine del 1962 [Lewisohn 2013].

Il libro si propone come autorevole interlocutore di quella variegata produzione, accademica e non, che nell'arco degli ultimi tre decenni ha profondamente cambiato il modo di guardare alla popular music, all'intersezione fra musicologia, sociologia della cultura di massa e dei consumi, storia della musica e storia culturale, sociale ed economica. E' una produzione, questa, nella quale un'attenzione per la storia mai disgiunta dalla materia musicale, sollecitata e praticata in origine soprattutto da musicologi e storici della musica particolarmente sensibili quali Richard Middleton o Franco Fabbri, ha progressivamente suscitato l'interesse anche di storici culturali e sociali. Nel caso dei Beatles ne sono emerse indagini di notevole valore, con poche eccezioni [McMillian 2014] non tradotte in italiano, su singoli aspetti della storia della band: dal genere, alla classe, alla razza, alla dimensione produttiva e industriale, ai fan, al rapporto con altri gruppi [Fasce 2017 e 2018a].

\section{UNA RICERCA CONCLUSA E UNA INIZIATA}

Con la piena consapevolezza dei limiti dovuti al fatto di non saper suonare né tanto meno leggere la musica, limiti ai quali naturalmente neppure il generoso aiuto fornitomi da musicologi e musicisti di vaglia ha potuto ovviare, ne La musica nel tempo ho provato a integrare la letteratura accademica e non accademica in una prospettiva di storia culturale su base narrativa. Ho provato, cioè, a mettere in valore nel cantiere beatlesiano le profonde conoscenze che continuano ad accumularsi sugli aspetti più disparati, musicali e non, dei «lunghi anni sessanta». E a usare fonti primarie a stampa, ma anche in parte inedite (ad esempio, materiali del Foreign Office o carte dei critici musicali dell'epoca), per fornire un'immagine, sintetica, ma il più documentata possibile, di un oggetto tanto iconico e mitico attraverso il filo conduttore della storia culturale della musica come pratica di lavoro, di consumo e di costruzione di identità. 
Con uno spirito affine sto impostando la ricerca sul tour italiano dei Beatles di cui parlo in queste pagine. Esse dunque costituiscono una prima lettura ragionata, a voce alta, delle schede sulla letteratura e sulle fonti primarie a stampa raccolte finora, base per un lavoro in profondità, su fonti orali e d'archivio, ancora in larga parte da fare. Occorre tener conto che finora in nessuna delle ricostruzioni complessive straniere la tournée italiana figura con un qualche rilievo. Obiettivo finale del lavoro sarà, sull'esempio delle ricerche internazionali intorno ad altre tournée dei Beatles [Zion], disegnarne l'andamento e l'impatto, sulla carriera del gruppo e sulla musica, la società e le culture giovanili del nostro paese. Nel tentativo di illuminare quella faccia musicale del «lungo '68» che anche a giudizio di quanti se ne sono autorevolmente occupati di recente resta ancora in larga parte inesplorata [Bergoglio 2018; Brusco 2018; Liperi 2018; Bertoncelli 2018].

\section{BEATLES ‘ 65}

Quando, nel giugno 1965, approdano in Italia, i Beatles sono da oltre un anno una merce globale, anche se di un globo confinato sostanzialmente al mondo di lingua inglese e nord-europeo. Fortuna, talento e l'aiuto decisivo di alcuni mediatori intergenerazionali (il manager Epstein, il produttore Martin, l'editore musicale James) non propriamente mainstream e inguaribilmente predisposti alla sperimentazione e al rischio hanno contribuito a proiettarli in vetta. Dal turbinio di serate, dischi e impegni di ogni genere (radio, tv, cinema) che li hanno assorbiti senza tregua per un paio d'anni e hanno fatto parlare di «beatlemania» sono uscite ridefinite per sempre le dimensioni e la natura delle esibizioni dal vivo (proiettate dai club e dai teatri verso le arene con oltre 10.000 spettatori) e si è imposto un inedito modello di gruppo vocal-strumentale con ambizioni di autonomia autoriale [Millard 2012; Lebovic 2017].

Il successo di pubblico che, nonostante le tante Cassandre che li vorrebbero finiti nello spazio di un mattino, non accenna a diminuire, è però solo uno degli elementi che compongono la complessa equazione sottesa al gruppo a metà decennio. Non meno rilevante è la tensione che agita il complesso mentre arriva a Milano. E' lo scarto, ormai palpabile, fra le sue crescenti aspirazioni artistiche, sotto l'incalzare di colleghi e rivali agguerriti e talentuosi, e la cruda routine delle esibizioni dal vivo. Ovvero quei concerti sui quali, è vero, il gruppo ha originariamente cementato se stesso e il suo rapporto con il pubblico, composto anche e soprattutto di ragazze teen, ma che ogni giorno di più si rivela una fonte di frustrazione. Perchè, visto lo stato decisamente arretrato della tecnologia di amplificazione e della struttura organizzativa di un settore cresciuto troppo in fretta, si risolve spesso in una bolgia nella quale i quattro, fra l'entusiasmo irrefrenabile degli spettatori, faticano a sentirsi fra loro e a farsi sentire [Fasce 2018a].

\section{LA CANZONE IN ITALIA}

\section{TRA PERSISTENZA E CAMBIAMENTO}

Qual è, in estrema sintesi, la situazione della musica leggera nella penisola quando vi sbarcano i Beatles? Sulla scorta di un'indagine Rai del dicembre 1964, il musicologo Paolo Prato la definisce «in bilico fra consuetudini radicate e voglia di cambiare» [Prato 2010, 317]. Lo sfondo è l'onda lunga, anche se momentaneamente appannata dalla cosiddetta «congiuntura», del «miracolo economico» e dell'affermarsi della discografia in quanto pivot del settore, rispetto al precedente, inveterato primato dell'editoria legata alla vendita degli spartiti. Cruciali, i nuovi veicoli distributivi e promozionali costituiti dai juke-box, arrivati nei bar nella seconda metà degli anni cinquanta e in 
nemmeno un decennio cresciuti da 500 a 20.000 unità, e dalle radio transistor, nel 1964 nelle mani di tre ragazzi su cinque (mentre solo uno su cinque possiede un giradischi e addirittura uno su venti un registratore). Messi insieme, questi strumenti contribuiscono a configurare un universo di inediti consumi giovanili incardinato attorno alla musica e al ballo, oltre che al cinema e all'abbigliamento [Gorgolini 2004]. Seguendo su una scala più ridotta (un terzo rispetto alla Gran Bretagna, un quarto alla Francia) dinamiche già evidenziate all'estero, soprattutto nel mondo angloamericano, il mercato discografico, con al centro sempre più i 45 giri, disegna una parabola di espansione particolarmente sostenuta fra il 1956 e il ' 60 , con un balzo da 12 a 20 milioni di dischi venduti, che arrivano addirittura a 30 nel ' 64 , almeno il 60 per cento assorbito dai giovani[3]. Risultato di questo articolato processo di pratiche che si intrecciano ai controversi e diseguali (su base territoriale, di classe e di genere), meccanismi di crescita e modernizzazione nazionali, la musica emerge sempre più chiaramente, scrive Prato, quale «sottofondo sonoro, divertimento e passatempo» della vita quotidiana. L'offerta che la innerva, tuttavia, appare ancora «assai povera», segnata dalle persistenze che si sovrappongono agli elementi di cambiamento [Prato 2010, 317-8].

\section{I "RITMICI MODERNI E MODERNISSIMI"}

Emerso già dagli anni a cavallo dei due decenni con una radicale riduzione dell'età degli esecutori che, nell'arco di appena un biennio (1958-60), scalzano dalle posizioni di testa della hit parade chi abbia più di trent'anni, a metà Sessanta il mutamento è rappresentato ancora dai giovani cantautori («melodici modernissimi» nel linguaggio Rai) quali il trentenne Paoli e il ventiseienne Giorgio Gaber, affacciatisi in quel biennio, e soprattutto dai giovani e giovanissimi «ritmici moderni e modernissimi», come li definiscono sempre i sondaggisti tv. Di chiara ispirazione statunitense, la musica ritmata ventenne degli ormai ampiamente affermati Adriano Celentano e Mina (rispettivamente, cantante maschile e femminile più venduti nel 1964-65 secondo «Musica e Dischi») e degli emergenti (nati cinque-sei anni dopo i primi due) Gianni Morandi e Rita Pavone, che li seguono a ruota, guida le classifiche. Ma nell'inchiesta Rai questa musica convive (e nella prassi, in diversi casi, vedi in specie Morandi, deve scendere a patti) con attori e stili tradizionali, ancora ben presenti nel settore e nel suo establishment, anche grazie alla rilevante vetrina fornita dall'ingessata programmazione radiotelevisiva. Esemplare in proposito il successo di «melodici moderni» in ascesa come l'adolescente Gigliola Cinquetti o consolidati quali il quasi quarantenne Claudio Villa, trionfatori nel 1964, rispettivamente, a Sanremo e nella competizione di punta tv Canzonissima. Cosa forse ancora più importante, gli impulsi al cambiamento devono fare i conti con la forte resistenza manifestata dal pubblico nei confronti dell'uso di lingue straniere. Un fenomeno, quest'ultimo, che, da un lato, spinge alla traduzione in lingua italiana dei brani, soprattutto dall'inglese, a opera degli stessi cantanti originali (un manipolo dei quali, con in testa i ventenni ex teen idol Paul Anka e Gene Pitney sempre nel 1964 ha trovato posto per la prima volta a Sanremo), e, dall'altro, incrementa la pratica delle cover per mano di artisti «indigeni» [Prato 2010].

\section{COMPLESSI}

\section{“TU VUOI CHE PARLI UN PO’ L'INGLESE"}

Attraverso una cover nel luglio del 1963, a cinque mesi dalla prima affermazione in testa alle classifiche nella madrepatria, una canzone dei Beatles si affaccia sulla scena italiana; scena sulla quale il gruppo era noto sino a questo momento solo agli sparuti ascoltatori di radio Luxembourg o alla minoranza che aveva accesso a dischi importati direttamente d'oltre Manica [Fabbri 2014]. E' la versione di Please please me, parto non proprio felice («Tu vuoi che parli un po' l'inglese») del 
navigato paroliere quarantenne, veterano della kermesse sanremese e di lì a poco autore per lo Zecchino d'Oro, Dante Panzuti, in arte Danpa. Giovanissimo (non ha ancora vent'anni) e pieno d'energia è invece l'esecutore, il bresciano Fausto Leali, leader del complesso vocal-strumentale i Novelty. Invero nel 1963 «complesso» è ancora un'espressione alquanto vaga, usata per indicare una variegata gamma di esperienze che riflettono la convulsa dialettica di tradizione e modernità in atto. Si va dal gruppo puramente vocale, a quello di accompagnamento di un solista, lungo una scala di generi e stili che abbraccia il liscio e la musica da night, sino al «Mersey beat» di cui riferisce «Musica e Dischi» nel settembre 1963 nel primo articolo italiano dedicato ai Beatles, in realtà una più ampia inchiesta su questa musica che, partendo da Liverpool, «sta elettrizzando» la scena giovanile inglese [Tomatis 2019; Brizi e Becker 2012].

Una lettura sistematica della stessa rivista nell'anno e mezzo che segue, sino alla tarda primavera del 1965, alle soglie del tour beatlesiano, consente di delineare il progressivo restringersi e precisarsi dell'espressione attorno alla formula prevalente del gruppo vocal-strumentale tipo Beatles, anche se ancora nel 1965-66 le classifiche di vendita del periodico mescolano sotto questa insegna il complesso vocale italiano dei Marcellos Ferial e i fab four[4]. Il processo definitorio corre parallelo a e si alimenta, in uno stretto rapporto reciproco, con il diffondersi della formula nella prassi musicale della penisola. Su entrambi i versanti, sulla scia degli Shadows, apripista prevalentemente strumentali d'inizio decennio, la band liverpulliana svolge un ruolo decisivo di esempio e punto di riferimento [Fabbri 2014; Tomatis 2019].

\section{“CIAO AMICI" E I BEATLES IN COPERTINA}

Segno della sua graduale visibilità nel panorama italiano è la copertina che le dedica nell'aprile 1964 il mensile «Ciao amici», la prima rivista giovanile a baricentro musicale che dal dicembre 1963 contribuisce a far da cassa di risonanza alla diffusione dei ritmi beat. Ricalcato dichiaratamente sin dal nome sul modello francese di «Salut les copains», il mensile suggella l'attenzione montante riservata al mercato teen. E mediante editoriali e una rubrica delle lettere colmi di ammiccamenti empatici e intenti a seguire e dettare mode, intercetta e sollecita impulsi e forme del sentire di una gioventù che le inchieste dell'epoca dipingono spesso come apatica e in cerca di un'identità [Alfassio-Grimaldi e Bertoni 1964; Piccone Stella 1998]. Diretta dal venticinquenne discografico e paroliere Luciano Giacotto, fra inserzioni pubblicitarie che promettono guerra ai brufoli e «unghie d'acciaio», propone un'identificazione generazionale incentrata sui modi di vita ruotanti attorno a quella che definisce la «nostra musica»: un perimetro artistico, produttivo ed esistenziale aperto al nuovo, ma entro i limiti di una contenuta polemica verso l'establishment «adulto» e «benpensante»; polemica che, come evidenzia il prevalere, nelle copertine del primo anno, dei cantanti di casa nostra, è comunque rispettosa del primato assegnato dalla sfera pubblica musicale nazionale ai prodotti autoctoni [Grispigni 1998; Volpi 2013]. Una delle cinque copertine del '64 riservate agli stranieri, l'unica dedicata a un gruppo, se la aggiudicano, al quinto numero, mentre Please please me è in classifica da alcuni mesi, i Beatles. Sulla falsariga di quanto accade all'estero, il giornale, spazio mediatico ibrido, commerciale e identitario, esalta il modello, musicale e comportamentale, "collettivo" dei quattro, sintetizzato nella formula Tutti per uno con la quale viene reso in italiano il titolo del loro primo film $A$ Hard Day's Night. E nel proporlo ai ragazzi come uno stile di vita da consumare e praticare, osserva il musicologo Jacopo Tomatis, rafforza in loro quel senso ancora indistinto di comunità e d'insieme che va emergendo soprattutto negli ambienti giovanili cattolici e nei gruppi informali dei «pari»su base amicale e scolastica [Tomatis 2019].

\section{LE BAND AUTOCTONE}


È un tema, questo, che meriterà di essere ripreso con analisi circostanziate sia sulla ricezione italiana della band, sia sulla funzione che possono aver svolto i «giovanili» - mediante la socializzazione da essi proposta attraverso la formazione di gruppi di ballo, ascolto e discussione di tematiche legate all'età, dalla scuola al sesso - nel passaggio dei loro lettori dalla dimensione aggregativa informale a quella più propriamente (o tradizionalmente) politica [Giachetti 2002]. Qui preme piuttosto sottolineare la proliferazione dei complessi autoctoni che, sulle orme dei fab four e di altre band, dagli Stones agli Animals, che animano l'incipiente «invasione britannica» dell'universo musicale mondiale, matura attorno alla metà del decennio. Nella primavera del '65 sono già abbastanza numerosi da guadagnarsi una sezione per sé, accanto a quelle dei «professionisti» e delle «giovani leve», nel raduno di cantanti organizzato da «Ciao amici» per i suoi lettori (l'ingresso è subordinato al possesso di un tagliando pubblicato dal giornale] al velodromo Vigorelli di Milano un mese prima della tournée beatlesiana)[5]. Se però guardiamo alle classifiche, sino a questo momento la presenza di gruppi è ancora molto rara. Il successo a mani basse decretato da «Musica e dischi» ai Beatles nella sezione «complessi» per il 1964 non riflette un'affermazione quantitativa di valore assoluto, ma pare piuttosto il segno di una presenza pionieristica di categoria, sostenuta da due soli 45 giri nella top ten e da un quarto posto come posizione massima raggiunta (anche le più generose statistiche compilate ex post dal giornalista Dario Salvatori, del resto, riconoscono alla band un solo primo posto su una presenza totale in classifica di 31 settimane nell'anno e mezzo compreso fra il lancio del loro primo disco e il giugno 1965) [De Luigi jr. 2007; Salvatori 1982].

\section{UN SUCCESSO TIEPIDO}

Come spiegare questo esito decisamente modesto rispetto a quello travolgente conseguito dal gruppo nel mondo anglosassone e nord-europeo? Le cronache dell'epoca e le ricostruzioni successive suggeriscono come possibili fattori anzitutto i residui autarchici e l'endemico problema della lingua, poi qualche esitazione del distributore locale, la Carisch, nel lancio del gruppo e forse il fatto che i suoi microsolchi arrivano sul nostro mercato in un anno, il 1964, che, se in termini assoluti segna il record dei 30 milioni di dischi venduti, rappresenta però anche una contrazione nel ritmo di crescita rispetto agli anni precedenti tale da spingere gli addetti ai lavori a parlare di «crisi». Di difficoltà col mercato, d'altronde, parla esplicitamente John Lennon in un'intervista rilasciata al giornalista Gianni Minà alla vigilia dello sbarco in Italia, nella quale dice che promuovere i dischi in modo da farli entrare stabilmente nella top ten costituisce la ragione principale del tour nella penisola [Fiore, Felcher e Colombi 2005; Buttava e Gentile 2007; Brizi e Becker 2012].

\section{CONCERTI DELLA TOURNÉE ITALIANA}

Mi concentro su quattro aspetti della tournée. Il primo è la natura assolutamente inedita dell'evento per il nostro paese. Il secondo è una breve esposizione del suo andamento con particolare riferimento ai risultati al botteghino. Il terzo sono le reazioni della carta stampata. Infine proverò ad accennare ai suoi effetti sulla presenza dei Beatles e dei gruppi che a essi si ispiravano nel panorama musicale della penisola e sui suoi fruitori, musicisti e non.

\section{LA PRIMA TOURNÉE AMERICANA}

Per affrontare la prima questione si impone uno sguardo sulla storia internazionale delle esibizioni dal vivo di cantanti e gruppi. E' uno sguardo necessariamente cursorio, per motivi di spazio e soprattutto perché la ricerca in materia, sia a livello internazionale, sia, a maggior ragione, da noi, 
risulta ancora ferma a uno stadio alquanto embrionale, nonostante alcuni recenti contributi di assoluto rilievo [Waksman 2009; Frith, Brennan, Cloonan e Webster 2009; Salvatore 2018]. Un dato che pare incontrovertibile è il fatto che a metà anni sessanta il settore, principalmente per effetto dei Beatles, sta cambiando pelle, con un improvviso incremento dei volumi cui non corrisponde però un'adeguata consapevolezza delle sfide tecnologiche e organizzative sottese al mutamento di scala in atto. La tournée americana del gruppo dell'estate 1964 è stata in questo senso cruciale. Anche se non si è riusciti a battere il record di 26.500 spettatori stabilito da Elvis Presley nel 1956, a Dallas, durante la Texas State Fair, nell'insieme, per dimensioni e impatto, tale tour, con una media di quasi 14.000 biglietti venduti per show, ha rappresentato uno spartiacque, nella breve storia dei Beatles e in quella ben più lunga delle esibizioni dal vivo in generale [Fasce 2018b].

\section{IL PIPER}

E in Italia? Al momento del tour il decennio sessanta ha visto avanzare tre fenomeni nuovi in questo campo. Li ripercorriamo in ordine decrescente dal punto di vista cronologico e crescente da quello delle dimensioni. Il più recente riguarda l'apertura a Roma nel febbraio 1965 del Piper, il primo club musicale indirizzato programmaticamente ai giovani. Avviato da un avvocato quarantenne giovanilista e con la vocazione dell'imprenditore dell'intrattenimento maturata attraverso la frequentazione dei club newyorkesi, invero l'esperimento risulta ben presto «anfibio» [Volpi 2013, 17]. I giovani infatti vi convivono con un pubblico più grande, composto di vip e non, in cerca di visibilità e sensazioni proibite nei ritmi frenetici rovesciati dalla pedana del locale dai Rokes, conterranei ed emuli dei Beatles da un anno in pianta stabile in Italia [Capuzzo 2003]. Il che non toglie che il grido di dolore («non ci imitano più») che le movenze «tribali» dei 1.500 scatenati spettatori-ballerini, in gran parte ragazzi, strappano alla cronista del giornale dell'UDI «Vie Nuove» in una vibrata denuncia dello «ye-ye» e dei suoi «falsi modelli » commerciali[6] sia una preziosa testimonianza del carattere eminentemente fisico che la partecipazione musicale giovanile va acquisendo [Tonelli 1998; Prato 2010] e di quella separatezza generazionale che ricorre come un refrain in tanti documenti dell'epoca [Casillo 2012; Socrate 2018].

\section{RADUNI MUSICALI DI “CIAO AMICI”}

L'anno prima scene di «isterismo collettivo», secondo la definizione che i principali quotidiani e periodici di casa nostra riservano a fenomeni del genere, in Italia e all'estero, hanno caratterizzato, complici le dimensioni sensibilmente più ampie, la comparsa dei raduni musicali organizzati da «Ciao amici». Ne sono protagonisti, introdotti da Mike Bongiorno, il quarantenne presentatore più popolare della Rai, in larga maggioranza cantanti solisti, quasi esclusivamente italiani (Celentano, Morandi, Leali), più alcuni stranieri, soprattutto francesi, che cantano comunque in italiano (Francoise Hardy), e alcuni complessi, con la sola presenza straniera degli italianizzati Rokes. Pubblico rumorosamente partecipante, i 7000 che affollano nell'aprile 1964 il Palazzetto dello Sport di Milano, i 25.000 del Palasport di Genova (comprese alcune migliaia costrette ad accontentarsi degli altoparlanti collocati fuori del palazzetto tutto esaurito) dell'ottobre successivo e i 7.500 ciascuno dei due pomeriggi del velodromo milanese Vigorelli del maggio 1965[7]. Da notare che prima di questi raduni l'evento più significativo in qualche modo a loro apparentabile come target ed entità era stato il Festival del rock milanese del maggio 1957, con 7000 persone che avevano «preso d'assalto [...] il Palazzo del Ghiaccio» cittadino [Merolla 2011, 125].

\section{IL CANTAGIRO}

Ancora maggiori sono i volumi riguardanti un terzo tipo di spettacolo dal vivo che ha esordito nell'estate del 1962 [De Carlo e Triviani 2017]. Come indica il nome, Cantagiro, è una specie di Giro d'Italia musicale partorito dal quasi quarantenne Ezio Radaelli, ex funzionario sindacale di 
sinistra riconvertito, attraverso la gestione del concorso per Miss Italia e gli uffici stampa di alcune case di produzione cinematografica, al mondo dello spettacolo. In un percorso a tappe sancito da giurie scelte sul posto la kermesse contrappone alla formula festivaliera di Sanremo («sinfonie melodiche, immobilismo dei cantanti sul palco, pubblico educato e distinto in sala») un'atmosfera materiata di movimento e di contatto diretto con le strade, le piazze, gli stadi [Giachetti, 93]. Se pure esplicitamente indirizzata a tutte le età, la carovana sembra esaltare lo spirito inquieto e la voglia di farsi sentire giovanili, come testimonia l'ampia presenza di ragazzi assiepati negli stadi e lungo le strade attraversate da questo spettacolo che alza ulteriormente la soglia delle presenze a una manifestazione di musica leggera, con una media di 25.000 persone a serata nel 1965[8].

\section{LA TOURNÉE ITALIANA: UNA SINTESI DI TUTTO QUESTO}

Il tour dei Beatles riassume in sé tutte e tre queste forme di spettacolo. Dal punto di vista delle sedi scelte, sull'asse Milano (velodromo Vigorelli) -Genova (Palasport) oscilla fra i raduni giovanili di «Ciao amici» e il Cantagiro. Da quello del target si colloca dalle parti del Piper e dei raduni del periodico per ragazzi. Ma, è bene rimarcarlo, per la prima volta porta questo target e quelle dimensioni a misurarsi con un solo artista collettivo, e di lingua straniera, anche se, com'è costume delle tournée internazionali, preceduto da una serie di "numeri", tutti italiani, ai quali è affidato il compito di riscaldare l'ambiente in attesa dell'attrazione principale.

L'eccezionalità dell'evento nel panorama musicale della penisola e l'ancora limitata presa dei suoi protagonisti sul piano discografico spiegano perché a organizzarlo sia una figura ardimentosa ed eccentrica dell'impresariato artistico e musicale dell'epoca, il quarantenne ebreo galiziano di nascita e milanese d'adozione Leo Wachter. Vittima in gioventù delle leggi razziali fasciste, incarcerato dal regime nel 1942 e poi partigiano nel Varesotto, nel dopoguerra Wachter si getta nel mondo dello spettacolo, costruendosi la fama di impresario aperto a ogni avventura, capace di portare in Italia il coro dell'Armata Rossa e i nomi migliori del jazz e dello swing, dalla Fitzgerald a Sinatra. Al momento di gestire la tournée dei Beatles, grazie a un contatto con Epstein, non esita di fronte al fatto che non hanno ancora sfondato nelle classifiche o bucato il video Rai, o agli avvertimenti di chi li dà per finiti, come tanti altri fenomeni della musica pop, nel giro di pochi mesi. Come già è accaduto in occasione della prima tournée americana, anche lui, come l'impresario newyorkese Sidney Bernstein, parla con Epstein in yiddish. Senza saperlo entra di diritto nel network, in costante espansione, di persone estrose e anticonvenzionali (da Epstein, a Martin, al regista cinematografico Dick Lester) che hanno fatto la fortuna dei Beatles e la propria mettendo allegramente da parte la paura di avventurarsi su terreni nuovi e accidentati [Catacchio 2007; Wachter 2010; Fiore, Felcher e Colombi, 2005].

\section{IL BOTTEGHINO}

Dell'eccezionalità dell'evento bisogna tener conto anche quando se ne esamina l'andamento, a cominciare dai risultati al botteghino. Ricordiamo che la tournée comprende tre tappe (Milano. Genova e Torino) e otto concerti (due al Vigorelli di Milano, due al Palasport di Genova, quattro al teatro Adriano di Roma). Secondo le cifre più attendibili, che si possono dedurre confrontando la pubblicistica e le ricostruzioni successive, ma che andranno verificate con l'esame puntuale dei borderò, per gli otto concerti sono stati strappati circa 47.000 biglietti. 5.000 di questi riguardano le esibizioni romane (la metà della capienza del teatro Adriano). Gli altri riguardano Milano (2526.000 spettatori, di cui 7.000 nello spettacolo pomeridiano e 19.000 in quello serale) e Genova (che invero, secondo alcune fonti giornalistiche, oscilla fra i 14 e i 15.000 spettatori, di cui 4-5.000 il pomeriggio e 10.000 la sera, mentre secondo Patrizia Wachter, figlia dell'impresario e a tutt'oggi depositaria dei borderò, la quota pomeridiana va alzata a 6.000) [Fiore, Felcher e Colombi, 2005, 45 e 72-3; Wachter 2010, 45]. Integrate dalle immagini e dai resoconti dei giornali italiani dell'epoca 
(che denunciano impietosamente vuoti profondi nei concerti pomeridiani, in specie quello genovese e quelli romani), le cifre paiono giustificare la conclusione di chi oggi definisce il tour, se non proprio un insuccesso, comunque un «successo non strepitoso» [Fiore, Felcher e Colombi, 2005, 86]. Ma ciò non deve impedirci di scavare ulteriormente sia sul lato di una sua più chiara contestualizzazione comparativa rispetto allo stato degli spettacoli dal vivo di musica leggera dell'epoca e alle possibilità e ai vincoli, di classe e di genere, economici e culturali, nell'accesso a questo tipo di consumi; sia su quello di uno sguardo in profondità e di un'etnografia critica dei concerti come riti collettivi.

\section{IL GIUDIZIO DEI MEDIA}

Lo stesso sguardo critico va applicato evidentemente alla questione di come il tour fu considerato dai media e in particolare dai giornali. Su questo punto il primo esame della stampa da me effettuato, sulla scorta degli utili lavori di sintesi giornalistica sinora apparsi in proposito, fa emergere un dato lampante, già segnalato dallo storico inglese Arthur Marwik in un suo classico studio sugli anni Sessanta nel mondo occidentale. Si tratta della profonda spaccatura che la tournée evidenziò fra la pubblicistica generalista mainstream e i «giovanili» [Marwick 1998, 467]. Il plurale si impone perché nel frattempo, proprio in occasione della tournéè, «Ciao amici», che da questo momento diventa quindicinale, è affiancato dal più ambizioso «Big».

Entrambi i periodici danno un giudizio entusiasta della tournée. Mentre, con poche eccezioni[9], quasi tutti i principali organi di stampa, anche quelli pure a tratti simpatetici come «L'Unità», rubricano il gruppo, secondo un modello discorsivo invalso a lungo anche in Gran Bretagna e negli Stati Uniti [Fasce 2018a] e che il frastuono che accompagna i concerti pare giustificare, prima di tutto come un fatto di costume (quattro zazzeruti), più che musicale [Bertoncelli 2012]. I Beatles sono considerati come un fenomeno che asseconda e incentiva gli istinti meno nobili dei giovani, ma che in Italia non ha attecchito e non può attecchire come nel mondo anglosassone[10]. E qui scatta negli osservatori un misto di eccezionalismo nazionalista e di stigmatizzazione di genere. Solo alcune ragazze «isteriche», si dice, si sono lasciate irretire, al punto da sfidare le forze dell'ordine, da questo fenomeno, irrimediabilmente «inglese», frutto, azzarda qualcuno, dell'eccesso di repressione del puritanesimo anglosassone che cerca poi compensazioni estreme in questa «ostentazione di effeminatezza»[11]. La conclusione dell'anonimo cronista del «Corriere della sera» allo spegnersi delle luci, dopo l'ultimo concerto romano, è dunque sollevata e lapidaria: voltare pagina, «occuparci d'altro, ritrovare la pace»[12].

\section{PRENDI LA CHITARRA E VAI}

Sappiamo che non è andata esattamente così. Ma siamo ancora lontani da un'adeguata ricostruzione dell'impatto del tour. Ci sono, però, alcuni elementi per avviare la discussione. Il primo dato riguarda la presenza beatlesiana nelle classifiche discografiche italiane. Anche se non toccherà mai i livelli raggiunti altrove, la curva di questa presenza segna un pronunciato miglioramento nell'anno e mezzo che segue al tour rispetto ai diciotto mesi precedenti. Secondo le stime di Salvatori, il gruppo triplica le settimane complessive in classifica e vi piazza due numeri 1 [Salvatori 1982]. Quelle più caute e, si direbbe, più puntuali di «Musica e Dischi» mostrano comunque un incremento non indifferente, suggellato nell'aprile 1966 dal primo brano, Michelle, in testa alla classifiche - nonché sesta canzone assoluta di quell'anno, anche se quantitativamente ben lontana dai principali successi italiani, ovvero la metà delle copie della hit di Little Tony Riderà e addirittura un quinto del Ragazzo della via Gluck di Celentano - e un consolidamento nella sezione, ancora minoritaria, ma in chiaro aumento, dei 33 giri[13]. 
Forse ancora più rilevante è la generale crescita di legittimazione dei complessi, stranieri e autoctoni, già in parte in corso, ma che la tournée beatlesiana con tutta probabilità ha incentivato [Tomatis 2019]. Basti pensare alla presenza, per quanto sfortunata e fallimentare, di Equipe 84, Renegades e Yardbirds a Sanremo a soli sette mesi dal tour, alla creazione di una sezione riservata ai gruppi al Cantagiro dell'estate successiva, alla graduale avanzata di band italiane nelle hit parade, alle crescenti segnalazioni di loro dischi su «Musica e Dischi», che co-organizza con l'impresa di amplificatori e servizi musicali Davoli-Krull un festival dei complessi italiani. I gruppi ancora alle prime armi come gli Stregoni di Franco Fabbri o i Gleemen di Maurizio Cassinelli serbano un vivido ricordo dei concerti beatlesiani e riconoscono l'influenza cruciale della band di Liverpool, rinnovata anche da quell'esperienza [Fabbri 2011; Ferrando 2016]. Né pare inverosimile che tra i 6000 nuovi complessi che si creano tra il 1965 e il '66 [Gorgolini 2004] ce ne siano almeno alcuni che, come accade all'estero, sono spinti a tentare quest'avventura dall'atmosfera di montante favore per il beat e i gruppi che si sprigiona a cavallo della visita beatlesiana. E ancora, vien da pensare che non sia un caso isolato quello del quattordicenne genovese Franco Bampi, futuro fisico, che, folgorato dalla esibizione del gruppo, decide di imparare a suonare la chitarra[14], in un anno, il '65-66, nel quale la vendita di chitarre, in ascesa costante, aumenta di otto volte [Pivato 2005].

\section{UNA DEMOCRAZIA DELLE RAGAZZE?}

Sempre dal concerto genovese viene una testimonianza sul rumore assordante, che al tempo stesso conferma e complica le osservazioni stigmatizzanti dei giornali. Il quindicenne futuro musicista Bob Callero ricorda di essersene andato anzitempo, infastidito dal frastuono che impediva di «sentire una nota» e dalle urla in un «continuo passaggio di barellieri che portavano via a braccia queste teorie di ragazze» [Ferrando 2016]. Non è una cosa nuova e la ritroveremo puntualmente nemmeno due mesi dopo in alcune testimonianze del concerto allo Shea Stadium di New York, dove viene polverizzato il record di Presley con un tutto esaurito da 55.600 persone. Anche qui i ragazzi, specie se già un po' pratici di musica, criticano aspramente le urla, soprattutto di ragazze, spesso più giovani [Schwense 2013]. In questo atteggiamento si confondono insensibilmente un fenomeno reale (le urla delle ragazze), un habitus di genere che contrappone la razionalità maschile all'irrazionalità femminile e quell'asimmetria di ruoli, sempre di genere, finora non adeguatamente esplorata sul piano storico dalla letteratura di popular music, che vede il rock e il beat a lungo dominio praticamente esclusivo, in quanto attività musicale, dei maschi [Rhodes 2005; Leonard M. 2007; Roberts 2014]. E' interessante perciò confrontare questo atteggiamento con i ricordi delle ragazze presenti allo stesso concerto genovese. «Non mi ricordo scene di isterismo», dicono. «Noi gridavamo e cantavamo. Non avevamo modo di sentire le grida degli altri». E aggiungono che se ne uscirono con la «sensazione molto bella di far parte di una grossa comunità di ragazze, di ragazzi», partecipi del «primo evento per i giovani» in cui «si cominciò a essere teenager» [Ferrando 2016]. Altre rivendicano con orgoglio, nel ricordo, il fatto che «in mezzo a tutto quel rumore», prodotto dal loro cantare, gridare, saltare, «non si sentiva quasi nulla»: «ce ne fregavamo», dicono, «noi volevamo solo vedere i Beatles»[15].

Non si può non pensare immediatamente alle tante testimonianze, raccolte, all'epoca e in seguito, dalle storiche che, occupandosi delle ragazze dello Shea, hanno scoperto come le teenager fossero lì non tanto per ascoltare, quanto per vedere i Beatles, esserci, gridare la loro gioia e il piacere di condividere uno spazio affettivo, fra loro, e, per quanto magari solo mediante l'aiuto di un binocolo, con i loro beniamini, in quella che si configurava come la materializzazione di una «comunità immaginata» [Leonard C. 2014]. Ma evidentemente solo il prosieguo della ricerca mi permetterà di dire se anche nel caso italiano si possa parlare, come hanno fatto tali studiose, degli embrioni di un'ostinata, rumorosa «democrazia delle ragazze» in miniatura che prende corpo in questi concerti, nel pieno di una pratica di consumo [Rohr 2017]. E se il piccolo, non eclatante tour del ' 65 nasconda per avventura uno snodo non del tutto secondario di quella dialettica di «generazioni» (o 
sub-generazioni) «a confronto» che gli storici vanno scoprendo nel cuore del «lungo '68» [Socrate 2018].

\section{RIFERIMENTI BIBLIOGRAFICI E AUDIOVISIVI}

- Alfassio Grimaldi U. e Bertoni I. 1964, I giovani degli anni sessanta, Bari: Laterza.

- Bergoglio F. 2018, I giorni della musica e delle rose. Rock, pop, jazz, soul, blues nel vortice del `68, Viterbo: Stampa Alternativa

- Bertoncelli R. 2012, 1965-66, la nascita del nuovo rock, Firenze: Giunti.

- Bertoncelli R. 2018, 1968. Soul e rivoluzione, Firenze: Giunti.

- Brizi F. e Becker M. 2012, The Beatles in Italy. Come li raccontava la stampa, Roma: Arcana. Brusco F., Revolution. Il '68 dei Beatles, Arcana: Roma.

- Buttafava U. e Gentile E. (acd) 2007, Arrivano i Beatles. Storie di una generazione, Milano: Skira

- Capuzzo P. 2003, Gli spazi della nuova generazione, in Idem (ed.), Genere, generazione e consumi. L'Italia degli anni Sessanta, Roma: Carocci.

- Casillo S., Una generazione d'emergenza. L'Italia della controcultura (1965-1969), Le Monnier, Milano 2012.

- Catacchio A. 2007, Leo Wachter, il Sig. Beatles, "Alias-il manifesto", 15 dicembre 2007

- De Carlo, G. C. e -Triviani, J., Il Cantagiro oggi: una storia, un'emozione, un 'opportunità,

- Milano: ABeditore, 2017.

- De Luigi jr. M., Beatles: la rivoluzione a scoppio ritardato, in Buttafava U. e Gentile E. (acd), Arrivano i Beatles. Storie di una generazione, Milano: Skira.

- Davies H. 1970, I Beatles, Milano: Longanesi (ed. or. 1968).

- Fabbri F. 2011, Album bianco. Diari musicali 1965-2000, Milano: il Saggiatore.

- Fabbri F. 2014, And the Bitt Went On, in Fabbri F. e Plastino G. (acd), Made in Italy, New York: Routledge.

- Fasce 2017, Beatles, storici e storia a cinquant'anni da Sgt. Pepper's, “Contemporanea”, 2.

- Fasce 2018a, La musica nel tempo. Una storia dei Beatles, Torino : Einaudi.

- Fasce F. 2018b, I Beatles e i luoghi dei lunghi anni Sessanta, in Dondi M. e Salustri S. (acd) Sessantotto. Luoghi e rappresentazioni di un evento mondiale, Milano: Unicopli.

- Ferrando E. 2016, A Fab day's Night. Le giornate genovesi dei Beatles, DVD autoprodotto, Genova 2016

- Fiore F. 1999, The Beatles in Italy vol. 1, Le Energie: Arcore.

- Fiore F., Felcher I. e Colombi M. 2005, I Beatles in Italia, Total print, Genova 2005

- Frith S., Brennan M., Cloonan M. e Webster E. (acd) 2009, The History of Live Music in Britain, Volume I: 1950-1967: From Dance Hall to the 100 Club, New York: Routledge.

- Giachetti, D. 2002, Anni Sessanta comincia la danza: giovani, capelloni, studenti ed estremisti negli anni della contestazione, Pisa: BFS.

- Gorgolini L. 2004, Un mondo di giovani. Culture e consumi dopo il 1950, in Sorcinelli P. (ed.), Identikit del Novecento, Roma: Donzelli.

- Grasso R. 1980, The Beatles in Italy. 1963-1980. Discografia italiana completata dal repertorio iconografico delle copertine. Catania: Dafni.

- Grispigni M., S'avanza uno strano lettore. La stampa giovanile prima del '68, in Ghione P. e Grispigni M. (acd), Giovani prima della rivolta, Roma: Manifestolibri.

- Lebovic S. 2017, "Here, There, and Everywhere”: The Beatles, America, and Globalization, 1964-1968, "Journal of American Studies", 1.

- Hertsgaard M. 2002, La musica e l'arte dei Beatles. A day in the life, Milano: Baldini \& Castoldi (ed. or. 1995). 
- Leonard C. 2014, How the Beatles and Their Fans Remade the World, New York: Arcade.

- Leonard M. 2007, Gender in the Music Industry: Rock, Discourse and Girl Power, Ashgate: London.

- Lewisohn M. 2013, Tune In. The Beatles All These Years, vol 1, London: Crown Archetype.

- Liperi 2018, Ribelli e ostinati. Voci e suoni del 68, Roma: Manifestolibri.

- Marwick A. 1998, The sixties : cultural revolution in Britain, France, Italy and the United States, c.1958-c.1974, New York: Oxford University Press.

- McMillian J. 2014, Beatles vs Stones, Roma-Bari: Laterza (ed. or. 2013).

- Merolla M. 2011, Rock'n' roll Italian Way. Propaganda americana e modernizzazione nell'Italia che cambia al ritmo del rock 1954-64, Roma: Coniglio.

- Millard A. 2012, Beatlemania. Technology, Business, and Teen Culture In Cold War America, Baltimore: Johns Hopkins University Press.

- Norman P. 1981, Shout! La vera storia dei Beatles, Milano: Mondadori.

- Piccone Stella 1998, Le inchieste sociali, in Ghione P. e Grispigni M. (acd), Giovani prima della rivolta, Roma: Manifestolibri.

- Pivato S. 2005, Bella ciao. Canto e politica nella storia d'Italia, Roma-Bari: Laterza.

- Prato P. 2010, La musica italiana. Una storia sociale dall'Unità a oggi, Roma: Donzelli.

- Rhodes L.L., Electric Ladyland: Women and Rock Culture, 1965-75, Philadelphia: University Press Pennsylvania, 2005.

- Rizzi C. 2009, The Beatles, Firenze: Giunti.

- Rohr N. 2017, Yeah yeah yeah: The sixties screamscape of Beatlemania, "Journal of Popular Music Studies", 2.

- Salvatore G. 2018, Il teatro musicale del rock. Avanguardie, frontmen, light-show, Viterbo: Stampa Alternativa.

- Salvatori D. 1982, Venticinque anni di hit-parade in Italia, Milano: Mondadori

- Schwense D. 2013, The Beatles At Shea Stadium: The Story Behind Their Greatest Concert, New York: North Shore Publishing.

- Socrate F. 2018, Sessantotto. Due generazioni a confronto, Roma-Bari: Laterza.

- Spitz B. 2006, The Beatles. La vera storia, Milano: Sperling \& Kupfer (ed. or.)

- Tomatis J. 2019, Storia culturale della canzone italiana, , Milano: il Saggiatore.

- Tonelli, Anna 1998, E ballando ballando. La storia d'Italia a passi di danza (1815-1996), Milano : F. Angeli.

- Torkelson Weber E.,The Beatles and the Historians. An Analysis of Writings About the Fab Four, Jefferson: McFarland.

- Volpi A. 2013, Musica, politica e carta stampata. Dal beat a Parco Lambro, Ospedaletto: Pacini.

- Wachter P. 2010, Papà Leo. I Beatles, la balena Jonas, il Ciak e altre magie: dietro le quinte di un grande impresario, Milano: Bompiani.

- Waksman, S. 2009, This Ain't The Summer of Love. Conflict and Crossover in Heavy Metal and Punk, Berkeley: University of California Press.

- Zion L, The impact of the Beatles on pop music in Australia: 1963-66, "Popular Music", 1987, 3 .

Note:

[1] In effetti c'è un terzo volume con questo titolo che ha per oggetto la discografia beatlesiana italiana. Vedi Grasso 1980. 
[2] Per la letteratura in proposito mi permetto di rinviare a Fasce 2018a. Devo a Riccardo Bertoncelli, Franco Fabbri, Paolo Prato, Gianfranco Salvatore e Franco Zanetti l'esempio delle loro ricerche e alcuni consigli decisivi per l'impostazione del mio lavoro.

[3] Dischi: seria flessione 64-65 dopo un traguardo di 30 milioni, "Musica \& Dischi”, n. 226, aprile 1965.

[4] Bilancio di un anno, "Musica \& Dischi”, n. 234, 15 dicembre 1965-15 gennaio 1966.

[5] Raduno di cantanti al Vigorelli, "Musica \& Dischi”, n. 227, maggio 1965.

[6] M. Maffei, Rivolta a 45 giri, "Noi donne”, 27 marzo 1965.

[7] C'eravamo tutti, "Ciao amici", maggio 1964, n. 5; Il grande raduno degli amici a Genova, "Ciao amici”, novembre 1964, n. 11.

[8] Rita Pavone ha vinto la quarta edizione del Cantagiro, "Il Secolo XIX", 11 luglio 1965.

[9] A. Bland, Nessuno è riuscito a sentire i Beatles fra il pubblico urlante del Vigorelli, "La Stampa", 25 giugno 1965.

[10] M. Manciotti, Bravi i Beatles al Palasport. Ma la folla non è impazzita,'Il Secolo XIX”, 27 giugno 1965.

[11] R. Tedeschi, Salutato con entusiasmo il debutto milanese del celebre quartetto, "L'Unità", 25 giugno 1965.

[12] C.L., Tepida l'accoglienza dei romani ai Beatles, "Corriere della sera", 27 giugno 1965.

[13] M. DeLuigi jr., A tu per tu con le cifre, "Musica \& dischi”, n. 246, 15 dicembre 1966-15 gennaio 1967.

[14] Franco Bampi, testimonianza nel programma televisivo « Genova e i Beatles», http://www.primocanale.it/video/terza-genova-e-i-beatles-107052.html sabato 09 febbraio 2019, ultimo accesso 20 febbraio 2019.

[15] Isola L., "Beatles: Genova come Liverpool”, tesina per il corso Magistrale di Storia Contemporanea, Università di Genova, a. a. 2014-15, inedita, in possesso dell'autore. 